lensity of the stream diminish as its length increases, its interlacing wreaths will give rise to a group of meteor-showers, more and more difficult to distinguish from each other, as their number becomes greater, until at last the condition of a meteor-belt so formed becomes that of innumerable meteorparticles revolving in orbits apparently indepenclent of each other, and intersecting each other in all possible directions within the general boundaries of the elliptic ring. The appearance presented by a meteor-group of this description, during its first encounters with the earth, will be a periodic starshower (like that of the November meteors), diverging, whenever it is visible, from a nearly exact and single radiant point. At the end of a certain number of cyclical retums, the star shower will be annually visible on a particular date, diverging from the same, or nearly from the same, radiant-point, but much less abundantly than at first; and a twin meteor-shower with a time of maximum, and a radiant point closely adjacent to the former ones will, at intervals, make its appearance with the original shower. This also, like the latter, after an equal lapse of time, will become annual ; and both diminishing together will present the appearance of a double meteor-shower, appearing simultaneously, or very nearly together, with a double or twin radiantpoint; while at intervals, a third meteor-shower, of the same general features as the previous two, will begin to be added to the group. Proceeding in this mamner, as the antiouity of the meteor-ring increases, the star-shower will resolve itself into a more or less well. defined group of slender streams, producing alternate short lulls, and flights of meteors from a great multiplicity of radiant points, contained within a limited region of diffuse or multiple radiation. The ordinary appearance of the star-shower on the nights of the gth and $\mathrm{rth}$ of August, answering very closely to the description of a meteorstream in an already far-advanced stage of its development, the much higher antiquity of the August than that of the November star-shower, already shown by its regular annual return, and by the ancient times in which it appears to have been recorded, must now also be regarded as satisfactorily confirmed by the frequently-recorded multiple, and more commonly observed diffuse character of its radiation. Among the star-showers of less ancient date, of which the November meteors appear to present a conspicuous example, Prof. Schiaparelli includes a meteorshower observed by Zezioli on October $\mathrm{x} 2$, and two others on November IO, 1868; one star-sllower on each of these dates radiating very exactly from points in the neighbourhood of the constellation Taurus, as well as the star-shower of October 18 , and 20,1864 and 1865 , the radiant point of which was very exactly marked in those years in Orion.

Continued observations of the best-known star-showers being calculated to afford such important information on the present conditions, and on the probable antiquity of their connection with the solar system, the committee propose to re examine the principal meteor-showers during the coming year, with suitable means for registering the meteors observed on each of the following dates, viz., August 9 to 11 , October 18 to 21 , November 13 to I5 (A.M.), December II to I3, I87 I, and January I to 3, and April 19 to 21,1872 , and to determine, as exactly as possible, the moments of maximum frequency, the rates of appearanc? ancl the principal points of radiation of the meteors visible on those days.

\section{THE LATE REV. W. V. HARCOURT'S RESEARCHES ON GLASS*}

THE subject of the preparation and optical properties of glasses of a great variety of chemical positions, formed, for nearly forty years, a favourite study with the late Mr. Harcourt. As stated in a report published in the British Association Reports for 1844 , some experiments on the subject were commenced in 1834 , which he was encouraged to pursue further by a request published in the fourth volume of the Transactions of the Association. A report on a gas furnace, the construction of which formed a preliminary inquiry, was published in the reports, but the results of the actual experiments on glass have never yet been published.

My own comnection with these experiments commenced at the meeting of the Association at Cambridge in 1862, when Mr. Harcourt placed in my hands some prisms formed of the glasses which he had prepared, to enable me to determine their character as to fluorescence. I was led iicidentally to observe the fixed lines of the spectra formed by them; and as I used sunlight

* Paper read by Prof. Stokes in Section A, British Association, 1871. which he had not found it convenient to employ, I was enabled to see further into the red and violet than he had done, which was favourable to a more accurate determination of the dispersive powers. This inquiry being in furtherance of the original object of the experiments, seemed far more important than that as to fluorescence, and the increased definiteness caused Mr. Harcourt to resume his experiments with the liveliest interest, an interest which he kept up to the last. Indeed, it was only a few days before his death that his last experiment was made. To show the extent of the inquiry I may mention that at least I 66 masses of glass were formed, and cut into prisms for measurement, each mass doubtless involving in many cases several preliminary experiments, besides discs and masses for other purposes.

It is well known how difficult it is, in working on a small scale, to make glass which is free from strize and imperfections of the kind. Of the first group of prisms, 28 in number, 10 only showed a few of the principal dark lines of the solar spectrum ; the rest had to be examined by the bright lines in artificial sources of light. These prisms seened to have been cut at random by the optician from the mass of glass furnished to him. Theory and observation alike showed that strize interfere comparatively little with an accurate determination of refractive indices when they lie in planes parpendicular to the edge of the prism. Accordingly, in the rest of the research the prisms were formed from the glass mass that came out of the crucible by cutting two planes passing through the same horizontal hine a little behind the surface, and inclined $22 \frac{1}{2}^{\circ}$ right and left of the vertical, and polishing the enclosed wedge of $45^{\circ}$. In the central portion of the mass the strize have a tendency to arrange themselves in nearly vertical lines by the operation of currents of convection, and by cutting in the manner described the most favourable direction of the strix is secured for a good part of the prism. This attention to the direction of cutting, combined no doub with increased experience in the preparation of glass, was attended with such good results that now it was quite the exception for a prism not to show the principal dark lines. Some of the latest prisms were almost equal to prisms of good optical glass.

On account of the difficulty of working with silicates, arising from difficult fusibility and the pasty character of the glasses, Mr. Harcourt's experiments were carried on with phosphates, combined in many cases with fluorides and sometimes with borates, tungstates, molybdates, and titanates. The glasses formed involved the elements potassium, sodium, lithium, barium, strontium, calcium, glucinium, aluminium, magnesium, manzanese, zinc, cadmium, tin, lead, thallium, nickel, chromium, uranium, bismuth, antimony, tungsten, molybdenum, titainium, vanadium, phosphorus, florine, boron, and sulphur. A very interesting subject of inquiry presented itself collaterally with the original object, namely, to ascertain whether glasses could be formed which would achromatise each other so as to exhibit no secondary spectrum, or a single glass which would form wit? crown and fint a combination achromatic in that sense. This inquiry presented considerable difficulties. The dispersion of a medium is small compared with its refraction, and if the dis. persion be regarded as a small quantity of the first order, the irrationality between the two media may be regarded as depend. ing on small quantities of the second order. If stria and imper fections of the kind present an obstacle to a very accurate determination of dispersive power, it will readily be understood that the errors of observation thus occasioned go far to swallow up the small quantities, in the observation of which the determination of irrationality depends. Accordingly little success attended the attempt to draw satisfactory conclusions as to irrationality from the direct observation of refractive indices; but by a particular mode of compensation, in which the experimental prism was achromatised by a prism built up of a combination of slender prisms of crown and flint, I was enabled to draw trustworthy conclusions as to the character, in this respect, of these prisms, which were good enough to show a few of the principal dark lines of the solar spectrum.

Theoretically any three different kinds of glass may be made to form a combination which shall be achromatic as to secondary as well as primary spectra; but for a long time little hope of a practical solution seemed to present itself. A prism containing molybdic acid was the first to give fair hopes of success. Mr. Harcourt warmly entered into the subject, which he prosecuted with un wearied zeal. The earlier molybdic glasses prepared were many of them rather deeply coloured, and most of them of a perish able nature. At last, after numerous experiments, molybdic glasses were obtained nearly free from colour, and permanent. Titanium had not yet been tried, and about this time a glass 
containing titanium was prepared. Titanic acid proved to be equal or superior to molybdic in its power of extending the blue end of the spectrum more than corresponds to the dispersive power of the glass; while in every other respect-freedom from colour, permanence of the glass, greater abundance of the element - it had a decided advantage; and a great number of titanic glasses were prepared, cut into prisms, and measured. Some of these led to the suspicion that boracic acid had an opposite effect to titanic, to test which Mr. Harcourt formed some simple borates of lead, with very varying proportions of boracic acid. These fully bore out the expectation; the terborate, for instance, which in dispersive power nearly agrees with flint glass, agrees on the other hand in the relative extension of the blue and red ends of the spectrum with a combination of about one part (by volume) of flint glass with two of crown.

By combining a negative (or concave) lens of terborate of lead with positive lenses of crown and flint, or else a positive lens of titanic glass with negatives of crown and flint, or a positive of crown and a negntive of low flint, achromatic triple combinations free from secondary dispersion might be formed, with. out encountering formidable curvatures, and by substituting at the same time a borate of lead for flint glass, and a titanic glass for crown, the curvatures might be a little further reduced.

There is no advantage in using three different kinds of glass rather than two, to form a fully achromatic combination, except that the latter course might require the two kinds of glass to be made to order, whereas with three we may employ for two of them the crown and flint of commerce. It is probable that enough titanium might be introduced into a glass to allow the glass to be properly achromatised by Chance's "light-flint."

In a triple combination of lenses the middle lens may be made to fit both the others, and be cemented. Terborate of lead, which is somewhat liable to tarnish, might thus be protected by being placed in the middle. Even if two kinds only of glass be ised it is desirable to divide the concave lens into two for the sake of diminishing the curvatures. On calculating the curvatures so as to destroy spherical as well as achromatic aberra. tion, and at the same time, to make the adjacent surfaces fit, very suitable forms were obtained with the data furnished by $\mathrm{Mr}$. Harcour't's glasses.

After encomntering great difficulties from stria, Mr. Harcourt at last succeeded in preparing discs of terborate of lead and of a titanic glass, of about 3 in. diameter, almost homogeneous, and with which it is intended to attempt the construction of an actual object glass, which shall give images free from secondary colour.

This notice extends to a greater length than I had intended, but still it gives only a meagre account of a research extending over so many years. It is my intention to draw up a full account for presentation to the scientific world in another way. I need but say that the small grant made to Mr. Harcourt for these researches has been expended over and over again, but it was his wish, in recognition of that grant, that the first notice of the results he obtained should be made to the British Associa. tion.

\section{THE BRITISH ASSOCIATION MEETING AT EDINBURGH}

\section{SECTION A.}

On the Recent and Coming Solar Eclipses, by J. N. Lockyer, F.R.S. The substance of this has already appeared in these pages.

Prof. Tait remarked, after the reading of Mr. Lockyer's paper, that the photograply which had been exhibited left no doubt in his mind that the greater part of the solar corona is produced in the earth's atmosphere. The rays are pretty obviously to be attributed to ice-crystals, and the various irregular protuberances sometimes seen may be due to germs and light particles blowin off from meteorites before they become incandescent, which, according to a beautiful investigation of Stokes, descend with extreme slowness towards the earth. This simple consideration is sufficient to show the utter absurdity of the sneers with which Sir W. Thomson's suggestion has been received, and to justify it as a scientific possibility-all it pretended to be.

On the Coming Solar Eclipse, by M. Janssen. In the discussion on these communications, Sir W. Thomson said he joined warmly in what Mr. Lockyer and M. Janssen had said. M. Janssen had asked that Britain should join France and Germany in their friendly struggle. There was a challenge from France and Germany, and it would be a disgrace to England if it did not accept that challenge, and do its best to beat its rivals in the struggle.

Report of the Committee for discussing Observations of Lunar Objects suspecticd of Change.

Mr. W. R. Birt, to whom the execution of the work was confided, read the report on behalf of the committee, consisting of Edward Crossley, Esq., and the Rev. T. W. Webb. The report stated that much attention having of late years been given to lunar objects, the purpose for which the committee had been appointed would be best carried out by confining the discussion to the observations of a small but well-known portion of the moon's surface; and as the spot plate had presented during the last two years a variety of interesting and important features which had been well observed, it had been chosen as the most likely to yield results contributing to the advancement of selenography. Time having permitted the discussion of the observations of the bright spots only, it was requested that a further grant of $20 \%$. should be placed at the disposal of the committee for the discussion of the observations of the streaks and markings on the floor which were intimately connected with the spots. Mr. Birt, in alluding to the work which he had executed on behalf of the committee, said that as his report was voluminous, he would content himself with a brief description of the results at which he had arrived. The area of Plato in which the spots exist measured about 2,700 square miles; as many as thirtyseven spots had been observed, but he wished it to be particularly understood that the whole had never been seen together; the greatest number observed on any one occasion was twenty-seven, the mean or average number being not more than eight. With the aid of diagrams drawn on the black board, he showed that the mean number seen at intervals of twelve hours of the lunisolar day varied during the progress of the day, so much so as to indicate that the number of spots visible at any given interval does not depend upon the angle at which the sun's light falls upon the floor of Plato. Some spots, he said, had been seer more frequently at abont sixty hours after sumrise upon the foor of Plato than at any other portion of the luni-solar day; the positions of these spots on the floor were pointed out, and it was remarked that they were situated in the western part of the crater, and they agreed in having been more frequently observed in August 1869, than at any other period of the observations. Other spots were observed more frequently at a later period of the observations than in August 1869 , and they had been seen more frequently at a later period of the day, or after the sun had passed the meridian. Daylight at the moon is equal to fourteen terrestrial days and nights. These facts Mr. Birt argued were incompatible with the assumption that variations of aspect were entirely dependent upon variations of illumination, and rather pointed to the existence of activity on the moon's surface, the exact nature of which required further observations to elucidate.

Report on Thernal Conductivity of IMetals, by Prof. Tait.

Prof. Tait, on the part of the committee appointed to report on this subject, drew attention to the relation that exists between electric and thermal conductivity of metals, and the effect on conductivity of a very small amount of impurity. $\mathrm{He}$ also sketched the apparatus made use of in the determination, and said that, as a new gasometer had been introduced, he had recommenced the whole of his investigations under better auspices and with hopes of very great accuracy.

On a New Stecm Gange, by Prof. Zenger.

Experiments on Vortex Rings, by H. Deacon.

Observations of Parallax of a Planetary Nebula, by D. Gill.

On a New Form of Constant Galjanic Battery, by Latimer Clark.

On a Method of Testing Subnerged Eilectric Cables, by C. F. Varley.

Description of Experiments made in the Physical Laboratory of the University of Glasgow to determine the Surface Conductivity for Heat of a Copper Ball, by Donald M'Farlane. The experiments described in this paper were made under the direction of Sir W. Thomson during the summers of 1865 and 1871 . A hot copper ball, having a thermo-electric junction at its centre, was suspended in the interior of a closed space kept at a constant temperature of about $16^{\circ} \mathrm{C}$. The other junction was kept at the temperature of the envelope; the circuit was completed through 